\title{
Article
}

\section{Isolated effects of caffeine and sodium bicarbonate ingestion on performance in the Yo-Yo test: A systematic review and meta-analysis}

Grgic, Jozo, Garofolini, Alessandro, Pickering, Craig, Duncan, Michael J, Tinsley, Grant M and Del Coso, Juan

Available at http://clok.uclan.ac.uk/29792/

Grgic, Jozo, Garofolini, Alessandro, Pickering, Craig, Duncan, Michael J, Tinsley, Grant $M$ and Del Coso, Juan (2019) Isolated effects of caffeine and sodium bicarbonate ingestion on performance in the Yo-Yo test: A systematic review and meta-analysis. Journal of Science and Medicine in Sport . ISSN 1440-2440

It is advisable to refer to the publisher's version if you intend to cite from the work. http://dx.doi.org/10.1016/j.jsams.2019.08.016

For more information about UCLan's research in this area go to http://www.uclan.ac.uk/researchgroups/ and search for <name of research Group>.

For information about Research generally at UCLan please go to http://www.uclan.ac.uk/research/

All outputs in CLoK are protected by Intellectual Property Rights law, including Copyright law. Copyright, IPR and Moral Rights for the works on this site are retained by the individual authors and/or other copyright owners. Terms and conditions for use of this material are defined in the policies page. 
1 Abstract

2 OBJECTIVES: To conduct a systematic review and a meta-analysis of studies exploring the

3 effects of caffeine and/or sodium bicarbonate on performance in the Yo-Yo test.

4 DESIGN: Systematic review/meta-analysis.

5 METHODS: A total of six databases were searched, and random-effects meta-analyses were performed examining the isolated effects of caffeine and sodium bicarbonate on performance

7 in the Yo-Yo test.

8 RESULTS: After reviewing 988 search records, 15 studies were included. For the effects of 9 caffeine on performance in the Yo-Yo test, the meta-analysis indicated a significant favoring of caffeine as compared with the placebo conditions $(p=0.022$; standardized mean difference $[\mathrm{SMD}]=0.17 ; 95 \% \mathrm{CI}: 0.08,0.32 ;+7.5 \%)$. Subgroup analyses indicated that the effects of caffeine were significant for the level 2 version of the Yo-Yo test, but not level 1. Four out of the five studies that explored the effects of sodium bicarbonate used the level 2 version of the Yo-Yo test. The pooled SMD favored the sodium bicarbonate condition as compared with the placebo/control conditions ( $p=0.007$; SMD: 0.36; 95\% CI: $0.10,0.63 ;+16.0 \%)$.

CONCLUSIONS: This review demonstrates that isolated ingestion of caffeine and sodium bicarbonate enhances performance in the Yo-Yo test. Given these ergogenic effects, the intake of caffeine and sodium bicarbonate before the Yo-Yo test needs to be standardized (i.e., either restricted or used in the same way before each testing session). Furthermore, the results suggest that individuals competing in sports involving intermittent exercise may consider supplementing with caffeine or sodium bicarbonate for acute improvements in performance. 


\section{Introduction}

The Yo-Yo intermittent recovery test was introduced in the 1990s and has gained substantial popularity as a method of estimating aerobic and anaerobic capacity in team-sports players. ${ }^{1}$ In undertaking the Yo-Yo intermittent recovery test, participants are required to run distances of $2 \times 20 \mathrm{~m}$ at progressively increasing speeds. Each $2 \times 20 \mathrm{~m}$ work block is interspersed with a 10-s period of jogging around a marker placed $5 \mathrm{~m}$ behind the finish line. The test ends when the individual cannot complete the run within the prescribed time, on two consecutive occasions. The outcome of this test is the total covered distance. This test is comprised of two sub-levels, level 1 and level 2, with level 2 starting at a higher initial speed and necessitating a large contribution from the anaerobic energy system. ${ }^{1}$ These tests are widely used in field settings as a practical method to: (a) determine current fitness status; and (b) prescribe training programs and explore their effectiveness. ${ }^{2}$

Caffeine and sodium bicarbonate are two supplements that have been shown to acutely enhance exercise performance. ${ }^{3}$ The effects of caffeine and sodium bicarbonate have also been explored in the context of Yo-Yo test performance, with equivocal findings. ${ }^{4-8}$ Some reported an increase in performance following the ingestion of either caffeine or sodium bicarbonate, while others report that performance in this test is unaffected by the use of these supplements. ${ }^{4-8}$ Differences between the studies such as the dose, ingestion timing, and training status may explain some of these discrepancies. ${ }^{9}$ Perhaps even more importantly, the studies conducted on the effects of sports supplements tend to be performed with small sample sizes. ${ }^{3}$ For example, one study that explored the effects of sodium bicarbonate on performance in the Yo-Yo test included only six participants. ${ }^{8}$ Therefore, there remains a possibility that some studies were statistically underpowered to observe significant effects, which may have resulted in increased probabilities of type II errors. Meta-analysis is a 
statistical method that can be used to overcome the limitation of underpowered studies because it allows combining of data from different cohorts to obtain a pooled estimated value.

To address the apparent discrepancies between individual studies two previous meta-analyses explored the effects of caffeine on performance in the Yo-Yo test. In the first analysis, Gonçalves Ribeiro et al. ${ }^{10}$ reported no significant effects of caffeine on performance in this test. However, the analysis included only two studies with a combined number of participants amounting to 31 . Such a small number of studies was included given that the authors limited their inclusion criteria to studies published between 2010 and 2015, even though no rationale was provided for this approach. Another limitation is that these authors included both crossover and between-group study designs. This may be relevant given that the inter-individual variation in responses to caffeine ingestion is not as well controlled in between-group designs as in cross-over trials. ${ }^{11}$

In another review, Salinero et al. ${ }^{12}$ reported that caffeine ingestion enhances performance in the Yo-Yo test by an effect size of 0.22 with a $95 \%$ confidence interval (CI) ranging from 0.00 to 0.44 . This meta-analysis included only four studies (pooled $n=57$ ) given that studies providing caffeine in doses lower than $2 \mathrm{mg} \cdot \mathrm{kg}^{-1}$ were not considered. Additionally, this review did not search through unpublished literature, which may have led to publication bias due to the "file drawer" syndrome. The "file drawer" syndrome suggests that studies with larger effect sizes are more likely to be published than those with small or non-significant effect sizes; therefore, including only published studies may present a source of bias in a given meta-analysis. ${ }^{13,14}$ Additionally, two studies ${ }^{4,15}$ were not included in the review by Salinero et al. ${ }^{12}$ and new studies on this topic have been published since this review. ${ }^{7,16}$ All these studies generated data for over 100 additional participants. Therefore, an updated meta- 
analysis that would include these studies could contain almost a threefold increase in the pooled sample size from the most recent meta-analysis ${ }^{12}$ thus increasing the veracity of the findings.

While the effects of caffeine have been explored using a meta-analytic approach, no previous meta-analyses explored the effects of sodium bicarbonate on performance in the Yo-Yo test. A meta-analysis exploring the effects of sodium bicarbonate on performance in this test is needed because: (a) the equivocal evidence on this topic presented in the literature; ${ }^{5,6,8}$ and, (b) the common anecdotal use of sodium bicarbonate by athletes. ${ }^{17}$ Therefore, the aim of this review was to explore the effects of caffeine and sodium bicarbonate on performance in the Yo-Yo testing using a meta-analysis and highlight the practical application of these findings.

\section{Methods}

For this review, peer-reviewed literature in the form of journal articles and unpublished literature in the form of a thesis, dissertations, or conference abstracts that investigated the effects of caffeine and/or sodium bicarbonate ingestion on performance in the Yo-Yo test was examined. The search for studies was conducted on April 14th, 2019. The following databases were searched: ERIC, PubMed/MEDLINE, SPORTDiscus, Open Access Thesis and Dissertations, Web of Science, and Scopus. Additionally, searches were performed within ResearchGate. In all of these databases, the following search syntax was used: ("caffeine" OR "supplement" OR "coffee" OR "ergogenic" OR "NaHCO3" OR "sodium bicarbonate") AND ("yo-yo" OR "yoyo" OR "yo yo"). Secondary searches were performed by screening the reference lists of the included studies, and by exploring the papers that cited the included studies. The search was performed independently by two authors (JG and AG). 
To be included in the review, studies were required to satisfy the following criteria: (1) published in English; (2) included apparently healthy humans as participants; and (3) employed a cross-over study design and explored the acute effects of caffeine and/or sodium bicarbonate on performance in any variant of the Yo-Yo test.

Two authors of the review (JG and AG) extracted the following data from the included studies: (1) author names and publication year; (2) sample size and the sample characteristics; (3) the variant of the Yo-Yo test used for the testing; and (4) main findings regarding the effects of caffeine and/or sodium bicarbonate on performance. In cases where the required data was presented in figures, the Web Plot Digitizer software was used for the extraction of raw values.

The PEDro checklist was used for assessing the quality of the included studies. ${ }^{18}$ This checklist has 11 items; these items refer to eligibility criteria, randomization and blinding of participants, blinding of assessors, the number of participants completing all testing sessions, and reporting of data for the key outcomes. While this checklist has 11 points, the first item is not included in the summary score, and therefore, the maximum number of points is 10 . Based on the summary scores, the studies were classified as being of excellent quality (9-10 points), good quality (6-8 points), fair quality (4-5 points) and poor methodological quality (<3 points). ${ }^{19-21}$ The quality assessment was conducted independently by two authors of the review ( $\mathrm{JG}$ and $\mathrm{AG})$.

The mean \pm standard deviation performance data extracted from the included studies were converted to standardized mean differences (SMDs) and their respective 95\% CIs. The following data are required to calculate SMDs: (1) Yo-Yo test performance mean \pm standard 
123 deviation of the caffeine/sodium bicarbonate and placebo/control trials, (2) total sample size,

124

125

126

127

128

129

130

131

132

133

134

135

136

137 and (3) inter-trial correlation. Inter-trial correlation was not presented in any of the included studies. As suggested in the Cochrane Handbook the following formula was used to estimate the correlation:

$$
r=\frac{S_{\text {placebo/control }}^{2}+S_{\text {treatment }}^{2}-S_{D}^{2}}{2 \cdot S_{\text {placebo } / \text { control }} \cdot S_{\text {treatment }}}
$$

S represents the standard deviation while $S_{D}$ is the standard deviation of the difference score, which was calculated as:

$$
S_{D}=\left(\frac{S_{\text {placebo/control }}^{2}}{n}+\frac{S_{\text {treatment }}^{2}}{n}\right)^{1 / 2}
$$

\section{One of the studies that explored the effects of caffeine used multiple caffeine doses; for this} study, SMDs and variances were calculated for each dose separately and the average values were used for the analysis. Two meta-analyses were performed: (1) for the effects of caffeine on performance in the Yo-Yo test; and (2) for the effects of sodium bicarbonate on performance in the Yo-Yo test. In the meta-analysis that focused on the effects of caffeine, a sensitivity analysis was performed by excluding one study in which caffeine was not ingested as it was provided in a mouth rinsing form. ${ }^{22}$ A subgroup analysis was performed for studies exploring the effects of caffeine on Yo-Yo intermittent recovery level 1 and for those exploring the level 2 variant of the test. In the meta-analysis for the effects of sodium bicarbonate, a sensitivity analysis was performed by excluding the only study that used the 
level 1 version of the test. SMD values of $<0.20,0.20-0.39,0.40-0.59,0.60-0.80$, and $>0.80$ were considered to represent trivial, small, medium, large, and very large effects, respectively. In each analysis, the $I^{2}$ statistic was used to explore heterogeneity with $I^{2}$ values of $<50 \%, 50$ to $75 \%$, and $>75 \%$ considered as low levels, moderate levels, and high levels of heterogeneity.

In addition to $95 \%$ CIs, 95\% prediction intervals (95\% PI) were calculated for both analyses by using the number of included studies in the meta-analysis, the pooled SMD, the upper limit of the $95 \% \mathrm{CI}$ and the tau-squared values. The 95\% PI denotes the range in which the effect size of a future study conducted on the topic will most likely be. Funnel plots asymmetry was explored only for the effects of caffeine given that there were less than 10 studies included in the analyses for sodium bicarbonate. Percent differences between supplement ingestion conditions and the placebo/control conditions were also calculated. The random-effects model was used for both analyses. The statistical significance threshold was set at $p<0.05$. All analyses were performed using the Comprehensive Meta-analysis software, version 2 (Biostat Inc., Englewood, NJ, USA).

\section{Results}

The total number of search results across all databases was 164. Of this number of search results, 21 full-text papers were read and 12 studies were included. The remaining documents were excluded based on the title or abstract. The secondary searches resulted in another 824 search results and in the inclusion of three additional studies. Therefore, in total, 15 studies were included; 13 studies were published as full-text manuscripts in peer-reviewed journals, one study was published as a conference abstract, and one study is a part of a master's thesis. . $^{4-}$ 8,13,15,16,22-28 Eleven studies explored the effects of caffeine, whereas five studies examined the 
171 effects of sodium bicarbonate on Yo-Yo test performance (Table 1). One study included examined the isolated effects of both caffeine and sodium bicarbonate.

For studies that explored the effects of caffeine, the pooled number of participants across all studies was 156 (21 females). The average sample size per study was 14 participants. In all of the included studies, the sample was comprised of athletes. The doses of caffeine in the studies ranged from $1 \mathrm{mg} \cdot \mathrm{kg}^{-1}$ to $6 \mathrm{mg} \cdot \mathrm{kg}^{-1}$. Five studies provided absolute doses of caffeine ranging from 200 to $500 \mathrm{mg}$. One study used a caffeine mouth rinsing form where $1.2 \%$ of the $25 \mathrm{ml}$ solution was caffeine. Timing of caffeine administration before exercise ranged from immediately before (one study), 5 minutes (two studies), 45 minutes (two studies), 50 minutes (one study), 60 minutes (four studies) and 70 minutes (one study) before exercise. Five studies used the intermittent Yo-Yo recovery test level 1 and six used the level 2 version.

The pooled number of participants for the studies that explored the effects of sodium bicarbonate was 46 (all males). The average sample size per study was 9 participants. Three studies included athletes as their study participants while two included recreationally active individuals. The doses of sodium bicarbonate in the studies ranged from $0.2 \mathrm{~g} \cdot \mathrm{kg}^{-1}$ to $0.4 \mathrm{~g} \cdot \mathrm{kg}$ ${ }^{1}$. Sodium bicarbonate was ingested 40 minutes pre-exercise (one study), 60 minutes (one study), and 90 minutes pre-exercise (two studies). One study used a protocol that included splitting up a dose of $0.4 \mathrm{~g} \cdot \mathrm{kg}^{-1}$ into five smaller doses taken at 90, 80, 70, 60, and 50 minutes pre-exercise. Four studies used the intermitted Yo-Yo recovery test level 2, with only one using the level 1 version.

Out of the eleven studies that explored the effects of caffeine on Yo-Yo test performance, eight were classified as being of excellent quality with two studies being classified as good 
methodological quality and one as fair methodological quality. Out of the five studies that explored the effects of sodium bicarbonate on Yo-Yo test performance, three were classified as being of excellent quality, one as good methodological quality and one study as being of fair methodological quality. No studies were classified as being poor methodological quality. Individual scores for the quality assessment can be found in Supplementary file 1.

The meta-analysis for the effects of caffeine included a total of ten studies given that in one study, the data required for the analysis was not presented and the authors did not provide the data upon written request. ${ }^{22}$ The main meta-analysis indicated a significant difference $(p=$ 0.022) between the caffeine and placebo conditions with the SMD favoring the caffeine condition (SMD: 0.17 ; $95 \%$ CI: $0.08,0.32$; percent change: $+7.5 \%$; $I^{2}: 28 \%$; $95 \%$ PI: -0.32 , 0.66; Figure 1). No funnel plot asymmetry was observed. In the sensitivity analysis in which the study that provided caffeine in a mouth rinsing form was excluded, the SMD values increased to 0.20 (95\% CI: $0.05,0.36 ; p=0.009$; percent change: $\left.+8.5 \% ; I^{2}: 26 \%\right)$. In the subgroup analysis for the level 1 Yo-Yo test the SMD was 0.02 (95\% CI: - $0.21,0.25 ; p=$ 0.880 ; percent change: $+2.5 \% ; I^{2}: 0 \%$ ). In the subgroup analysis for the level 2 Yo-Yo test the SMD was 0.31 (95\% CI: $0.12,0.51 ; p=0.002$; percent change: $\left.+14.4 \% ; I^{2}: 11 \%\right)$.

\section{The meta-analysis for the effects of sodium bicarbonate indicated a significant difference $(p=$} 0.007) between the sodium bicarbonate and placebo/control conditions. The pooled SMD favored the sodium bicarbonate condition (SMD: 0.36; 95\% CI: 0.10, 0.63; percent change: $+16.0 \% ; I^{2}: 14 \%$; $95 \%$ PI: $-0.61,1.33$; Figure 2 ). In the sensitivity analysis in which the study that used the level 1 Yo-Yo test version was excluded, the SMD values increased to 0.39 (95\% CI: $0.08,0.70 ; p=0.013$; percent change: $\left.+17.5 \% ; I^{2}: 25 \%\right)$. 


\section{Discussion}

222 This review reports that isolated ingestion of caffeine and sodium bicarbonate enhances performance in the Yo-Yo test. Both supplements seem to produce moderate performanceenhancing effects. Due to these acute ergogenic effects, the intake of caffeine and sodium bicarbonate before the Yo-Yo test needs to be standardized. The results also suggest that individuals competing in sports involving intermittent exercise may consider supplementing with caffeine or sodium bicarbonate for acute improvements in performance.

This meta-analysis adds further evidence that caffeine ingestion enhances performance in field-based tests of fitness. These results are in line with prior work in the area, most of which is based on tests performed in the laboratory. ${ }^{14}$ Caffeine's ergogenic effect is likely related to its binding to adenosine receptors. ${ }^{29}$ Caffeine has a similar structure to adenosine and therefore, when ingested, caffeine binds to $\mathrm{A}_{1}$ and $\mathrm{A}_{2 \mathrm{~A}}$ receptors, ultimately blunting the fatiguing effects of adenosine. As a result, acute caffeine ingestion may reduce perceived effort and increase physical performance. Caffeine ingestion may also enhance motor unit recruitment, thus leading to more forceful muscle contractions. ${ }^{30,31}$ These mechanisms might explain why the pooled SMD increased when the study that provided caffeine in the mouth rinsing form was excluded..$^{22}$ After excluding the study that utilized caffeine mouth rinsing, the SMD increased from 0.17 to 0.20 . Specifically, caffeine provided in this form does not increase plasma caffeine concentration ${ }^{32}$ which is a likely prerequisite for an ergogenic effect of caffeine.

Due to a small number of included studies, previous meta-analyses did not examine if the effects of caffeine differ between level 1 and level 2 versions of the Yo-Yo test. These 
subgroup analyses were performed in the present review and indicated that the effects of caffeine were significant only for the level 2 version. Such findings may suggest that caffeine is more effective in tests with a greater contribution from the anaerobic energy system. ${ }^{1}$ These results were obtained from studies that examined the effects of caffeine on either level 1 or level 2 versions of the test. However, given the inter-individual variation in responses to caffeine ingestion, ${ }^{11,33,34}$ future studies may consider comparing the effects of caffeine on performance in the level 1 and level 2 version of the test in the same group of participants.

Based on the results of this review, sodium bicarbonate is effective for acute increases in YoYo test performance. Sodium bicarbonate may elicit its ergogenic effects by: (a) acutely increasing blood bicarbonate and leading to a greater efflux of hydrogen ions $\left(\mathrm{H}^{+}\right)$and lactate out of the active muscles and into the circulation; and subsequently, (b) maintenance of intramuscular $\mathrm{pH} .{ }^{35,36}$ Following acute sodium bicarbonate ingestion and subsequent increase in blood bicarbonate concentration, the resultant $\mathrm{pH}$ gradient between the intracellular and extracellular environments favors the efflux of $\mathrm{H}^{+}$from the exercising muscle to blood, aiding intracellular $\mathrm{pH}$ regulation and reducing fatigue. ${ }^{35,36}$

While no previous meta-analysis examined the effects of sodium bicarbonate on Yo-Yo test performance, other meta-analyses have focused on different aspects of exercise performance. For example, Matson and $\operatorname{Tran}^{37}$ combined studies that explored the effects of sodium bicarbonate on various exercise tests, with some lasting over 30 minutes and others being of very short duration, and maximal intensity (e.g., 10-second "all-out" sprints). The pooled ergogenic effect size of sodium bicarbonate was 0.44 . The magnitude of the effect is comparable to the effect size observed herein, even though an argument can be made that the 
pooling of vastly different exercise tests (with different physiological demands) in the analysis by Matson and Tran ${ }^{37}$ might have been a methodological limitation. A meta-analysis by Carr et al. ${ }^{38}$ reported an ergogenic effect of acute sodium bicarbonate ingestion that amounted to $1.7 \%$. The performance-enhancing effect in the analysis by Carr et al. ${ }^{38}$ was small likely because it included many of the older studies that used protocols in which all participants ingest sodium bicarbonate around 60 to 90 minutes before exercise..$^{9,39,40}$ However, in recent years, studies have started using individualized time to peak blood bicarbonate protocols, and they generally report greater effect sizes. ${ }^{9,39,40}$ The majority of studies included in the meta-analysis utilized performance tests lasting up to 30 seconds (i.e., much shorter than the average duration of the Yo-Yo test), which limits further comparison of the results. Nonetheless, this review reinforces the suggestion of the International Olympic Committee that acute sodium bicarbonate ingestion enhances short-term high-intensity exercise performance. ${ }^{3}$

The results presented in this review highlight the need for standardizing caffeine and sodium bicarbonate intake before the Yo-Yo test. In other words, supplementation with caffeine or sodium bicarbonate should be either restricted or used in the same way before each testing session. If their use is not standardized, some individuals may ingest caffeine or sodium bicarbonate before the testing and possibly experience a supplement-induced improvement performance in the Yo-Yo test. This may be especially important to control when using the Yo-Yo test for the evaluation of the effectiveness of a given training program. Additionally, standardization of caffeine and sodium bicarbonate intake may be important for studies that focus on the reliability of the Yo-Yo test. ${ }^{41}$ If not standardized, ingestion of these supplements may lead to improvements in performance by small to moderate effects (i.e., pooled SMDs in 
293

294

295

296

297

298

299

300

301

302

303

304

305

306

307

308

309

310

311

312

this review ranged from 0.17 to 0.39 ); if not standardized, this improvement may affect the validity of the data and lead to incorrect interpretation of the results.

One additional value of these substances is that their ingestion may improve performance in training sessions or competitions with similar energy demands as the Yo-Yo test. This may be especially relevant in soccer given that Krustup et al. ${ }^{42}$ observed that the performance in the Yo-Yo test is significantly correlated $(r=0.81)$ with the number of high-intensity running actions performed at the end of each half of a game. These results suggest that caffeine or sodium bicarbonate ingestion may even improve performance directly during sports competitions. These supplements may also have the potential to enhance training responses and adaptations; albeit, future long-term studies are needed to establish such effects. Still, while the Yo-Yo test is valid for determining an individual's capacity to perform repeated exercise ${ }^{1}$ future studies may consider exploring the effects of caffeine and sodium bicarbonate using more specific team sport stimulations. For example, one study explored the effects of caffeine while using a performance test simulating physical and skill demands of a rugby union game which included seven circuits in each of two 40-min halves with a 10-min half-time rest. ${ }^{43}$

Using the PEDro checklist, the included studies are generally sound from a methodological quality perspective. Nonetheless, several included studies used a single-blind protocol which offers evidence of lower that the use of the "gold standard" double-blind study design. Of the studies that blinded the participants, only two ${ }^{16,28}$ explored the effectiveness of this blinding by asking the participants to indicate which condition was the placebo and which the caffeine/sodium bicarbonate one. This limitation needs to be addressed in future studies given 
that correct supplement identification may impact exercise outcomes and therefore lead to bias in the results. ${ }^{44}$

The main limitation is that some of the studies included in the meta-analysis on the effects of sodium bicarbonate used a placebo condition as the comparison and other employed a nonsupplement, control trial. This methodological aspect may have affected the results given that the act of ingesting a capsule (even though it does not contain an ergogenic compound) can produce improvements in performance due to the placebo effect. However, the largest improvement in performance following sodium bicarbonate ingestion was in a study ${ }^{26}$ that used a placebo vs. sodium bicarbonate comparison (SMD: $0.93 ;+30 \%$ ) which may suggest that this limitation may not be particularly impactful in this specific context (even though it needs to be stated). Additionally, it needs to be mentioned that only one ${ }^{25}$ of the five studies that explored the effects of sodium bicarbonate used a sodium-matched placebo comparison. Given that there are cases in which sodium can also be ergogenic, ${ }^{45}$ future studies on this topic should consider adding a condition with an equimolar amount of salt to the sodium bicarbonate condition to isolate the effects of bicarbonate. ${ }^{46}$

In the included studies, caffeine and sodium bicarbonate were provided in isolation. Given that both supplements appear to be ergogenic, future work is needed to explore whether their combined ingestion provides any additive benefits. It is possible that the combination of these supplements would provide even greater effects because acute ingestion of caffeine and sodium bicarbonate enhances performance through different physiological mechanisms. As stated previously, caffeine's ergogenic effects are explained by its tendency to bind to adenosine receptors while sodium bicarbonate elicits its ergogenic effect through $\mathrm{pH}$ 
regulation. While there are several studies conducted on this topic, future work is needed to provide greater clarity on the issue of supplement interactions. ${ }^{35}$ This area may be particularly relevant for athletes given that athletes often ingest more than one supplement, ${ }^{47}$ and that caffeine is often ingested as part of a multi-ingredient pre-workout supplement, or energy drink. Future studies are needed to explore optimal doses of caffeine and sodium bicarbonate that have the largest effect on performance while producing the least side-effects. ${ }^{48}$ In addition to doses, future work is need on the optimal timing of ingestion. Timing of ingestion may be especially important to investigate when it comes to sodium bicarbonate given that there is very large inter-individual variability in responses to sodium bicarbonate ingestion, ${ }^{9,39,40}$ and the timing of ingestion is also affected by the mode of delivery. ${ }^{49}$

\section{Conclusion}

The results of the present review indicate that isolated caffeine and sodium bicarbonate ingestion enhances performance in the Yo-Yo test. Given these acute performance-enhancing effects, the intake of caffeine and sodium bicarbonate before the Yo-Yo test needs to be standardized (i.e., either restricted or used in the same way before each testing session). Also, the results suggest that these substances may improve performance during exercise tasks with similar energy demands to the Yo-Yo test and athletes competing in intermittent sports may consider supplementing with caffeine or sodium bicarbonate for acute increases in performance. 
361 Conflicts of interest

362 None 


\section{References}

1. Bangsbo J, Iaia FM, Krustrup P. The Yo-Yo intermittent recovery test : a useful tool for evaluation of physical performance in intermittent sports. Sports Med 2008; 38(1):37-51.

2. Schmitz B, Pfeifer C, Kreitz K, et al. The Yo-Yo intermittent tests: a systematic review and structured compendium of test results. Front Physiol 2018; 9:870.

3. Maughan RJ, Burke LM, Dvorak J, et al. IOC consensus statement: dietary supplements and the high-performance athlete. Br J Sports Med. 2018; 52(7):439-455.

4. Mohr M, Nielsen JJ, Bangsbo J. Caffeine intake improves intense intermittent exercise performance and reduces muscle interstitial potassium accumulation. $J$ Appl Physiol 2011; 111(5):1372-1379.

5. Krustrup P, Ermidis G, Mohr M. Sodium bicarbonate intake improves high-intensity intermittent exercise performance in trained young men. J Int Soc Sports Nutr 2015; $12: 25$.

6. Cholewa JM, Grannis DJ, Jaffe DA, et al. The effects of sodium bicarbonate supplementation on a soccer specific conditioning test in division III soccer players. $J$ Trainology 2015; 4(1):19-24.

7. Ellis M, Noon M, Myers T, et al. Low doses of caffeine: enhancement of physical performance in elite adolescent male soccer players. Int J Sports Physiol Perform doi: 10.1123/ijspp.2018-0536

8. Tanaka S, Yamaguchi D, Igawa S. Effect of low-dose sodium bicarbonate supplementation on intermittent endurance performance. Food Nutr Sci 2018; 9:13161326. 
9. Heibel AB, Perim PHL, Oliveira LF, et al. Time to optimize supplementation: modifying factors influencing the individual responses to extracellular buffering agents. Front Nutr 2018; 5:35.

10. Gonçalves Ribeiro B, Pontes Morales A, Sampaio-Jorge F, et al. Acute effects of caffeine intake on athletic performance: a systematic review and meta-analysis. Rev Chil Nutr 2017; 44(3):283-291.

11. Pickering C, Kiely J. Are the current guidelines on caffeine use in sport optimal for everyone? Inter-individual variation in caffeine ergogenicity, and a move towards personalised sports nutrition. Sports Med 2018; 48(1):7-16.

12. Salinero JJ, Lara B, Del Coso J. Effects of acute ingestion of caffeine on team sports performance: a systematic review and meta-analysis. Res Sports Med 2019; 27(2):238256.

13. Ranchordas M, Kenzie, J. Effect of carbohydrate only and carbohydrate plus caffeine co-ingestion on a battery of reliable soccer-specific tests. Int J Sport Nutr Exerc Metab $2013 ; 23: 1-15$.

14. Grgic J, Grgic I, Pickering C, et al. Wake up and smell the coffee: caffeine supplementation and exercise performance - an umbrella review of 21 published meta-analyses. Br J Sports Med. 2019. doi: 10.1136/bjsports-2018-100278

15. Muro NI, Parada M. Effect of caffeine on aerobic endurance performance. Mexican J of Med Res 2016; 4:8.

16. Ranchordas MK, Pratt H, Parsons M, et al. Effect of caffeinated gum on a battery of rugby-specific tests in trained university-standard male rugby union players. J Int Soc Sports Nutr 2019; 16:17.

17. Burke LM. Practical considerations for bicarbonate loading and sports performance. Nestle Nutr Inst Workshop Ser 2013; 75:15-26. 
18. Maher CG, Sherrington C, Herbert RD, et al. Reliability of the PEDro scale for rating quality of randomized controlled trials. Phys Ther 2003; 83(8):713-721.

19. Grgic J. Caffeine ingestion enhances Wingate performance: a meta-analysis. Eur J Sport Sci 2018; 18(2):219-225.

20. Grgic J, Pickering C. The effects of caffeine ingestion on isokinetic muscular strength: A meta-analysis. J Sci Med Sport 2019; 22(3):353-360.

21. Grgic J, Trexler ET, Lazinica B, et al. Effects of caffeine intake on muscle strength and power: a systematic review and meta-analysis. J Int Soc Sports Nutr 2018; 15:11.

22. Dolan P, Witherbee KE, Peterson KM, et al. Effect of carbohydrate, caffeine, and carbohydrate + caffeine mouth rinsing on intermittent running performance in collegiate male lacrosse athletes. J Strength Cond Res 2017; 31(9):2473-2479.

23. Abian-Vicen J, Puente C, Salinero JJ, et al. A caffeinated energy drink improves jump performance in adolescent basketball players. Amino Acids 2014; 46:1333-1341.

24. Burke NR. Effects of caffeine supplementation on women's national league soccer players' performance. Master's thesis. 2016. University of Chester.

25. Dixon H, Baker CE, Baker JS, et al. Sodium bicarbonate ingestion improves Yo-Yo intermittent recovery test 1 performance: a randomized crossover trial. Nutr Diet Suppl 2017; 9:23-27.

26. Marriott M, Krustrup P, Mohr M. Ergogenic effects of caffeine and sodium bicarbonate supplementation on intermittent exercise performance preceded by intense arm cranking exercise. J Int Soc Sports Nutr 2015; 12:13.

27. Pettersen SA, Krustrup P, Bendiksen M, et al. Caffeine supplementation does not affect match activities and fatigue resistance during match play in young football players. J Sports Sci 2014; 32(20):1958-1965. 
28. Ranchordas MK, King G, Russell M, et al. Effects of caffeinated gum on a battery of soccer-specific tests in trained university-standard male soccer players. Int J Sport Nutr Exerc Metab 2018; 28(6):629-634.

29. McLellan TM, Caldwell JA, Lieberman HR. A review of caffeine's effects on cognitive, physical and occupational performance. Neurosci Biobehav Rev 2016; 71:294-312.

30. Black CD, Waddell DE, Gonglach AR. Caffeine's ergogenic effects on cycling: neuromuscular and perceptual factors. Med Sci Sports Exerc 2015; 47(6):1145-1158.

31. Grgic J, Mikulic P, Schoenfeld BJ, et al. The influence of caffeine supplementation on resistance exercise: a review. Sports Med 2019; 49(1):17-30.

32. Doering TM, Fell JW, Leveritt MD, et al. The effect of a caffeinated mouth-rinse on endurance cycling time-trial performance. Int J Sport Nutr Exerc Metab 2014; 24(1):90-97.

33. Sabol F, Grgic J, Mikulic P. The effects of three different doses of caffeine on jumping and throwing performance: a randomized, double-blind, crossover study. Int J Sports Physiol Perform 2019. doi: 10.1123/ijspp.2018-0884.

34. Grgic J, Mikulic P. Caffeine ingestion acutely enhances muscular strength and power but not muscular endurance in resistance-trained men. Eur J Sport Sci 2017; 17(8):1029-1036.

35. Burke LM. Practical issues in evidence-based use of performance supplements: supplement interactions, repeated use and individual responses. Sports Med 2017; 47(1):79-100.

36. Lancha Junior AH, Painelli Vde S, Saunders B, et al. Nutritional strategies to modulate intracellular and extracellular buffering capacity during high-intensity exercise. Sports Med 2015; 45(Suppl 1):S71-81. 
37. Matson LG, Tran ZV. Effects of sodium bicarbonate ingestion on anaerobic performance: a meta-analytic review. Int J Sport Nutr 1993; 3(1):2-28.

38. Carr AJ, Hopkins WG, Gore CJ. Effects of acute alkalosis and acidosis on performance: a meta-analysis. Sports Med 2011; 41(10):801-814.

39. Gough LA, Deb SK, Sparks SA, et al. Sodium bicarbonate improves $4 \mathrm{~km}$ time trial cycling performance when individualised to time to peak blood bicarbonate in trained male cyclists. J Sports Sci 2018; 36(15):1705-1712.

40. Gough LA, Deb SK, Sparks AS, et al. The reproducibility of blood acid base responses in male collegiate athletes following individualised doses of sodium bicarbonate: a randomised controlled crossover study. Sports Med 2017; 47(10):21172127.

41. Grgic J, Oppici L, Mikulic P, et al. Test-retest reliability of the Yo-Yo test: a systematic review. Sports Med 2019. doi: 10.1007/s40279-019-01143-4

42. Krustrup P, Mohr M, Ellingsgaard H, et al. Physical demands during an elite female soccer game: importance of training status. Med Sci Sports Exerc 2005; 37(7):12421248.

43. Stuart GR, Hopkins WG, Cook C, et al. Multiple effects of caffeine on simulated highintensity team-sport performance. Med Sci Sports Exerc 2005;37(11):1998-2005.

44. Saunders B, de Oliveira LP, da Silva RP, et al. Placebo in sports nutrition: a proof-ofprinciple study involving caffeine supplementation. Scand J Med Sci Sports 2017; 27(11):1240-1247.

45. Del Coso J, González-Millán C, Salinero JJ, et al. Effects of oral salt supplementation on physical performance during a half-ironman: A randomized controlled trial. Scand J Med Sci Sports 2016; 26(2):156-164. 
484

485

486

487

488

489

490

491

492

46. Bishop D, Claudius B. Effects of induced metabolic alkalosis on prolonged intermittent sprint performance. Med Sci Sport Exerc 2005; 37(5):757-767.

47. Bishop D. Dietary supplements and team-sport performance. Sports Med 2010; 40(12):995-1017.

48. Pickering C, Grgic J. Caffeine and exercise: what next? Sports Med 2019; 49(7):10071030.

49. Hilton NP, Leach NK, Sparks SA, et al. A novel ingestion strategy for sodium bicarbonate supplementation in a delayed-release form: a randomised crossover study in trained males. Sports Med Open 2019; 5(1):4. 